\title{
Salud Bucal y Enfermedades no transmisibles en pacientes de un centro de enseñanza universitaria del área Salud, Montevideo-Uruguay. Parte 2
}

\author{
Oral Health and Non communicable Diseases in patients of a higher \\ education institution, Montevideo, Uruguay 2016. Part 2
}

Saúde bucal e doenças não transmissíveis em pacientes de uma instituição de ensino superior, Montevidéu, Uruguai 2016.Parte 2

\author{
Susana Margarita Lorenzo-Erro ${ }^{1}$ (D) 0000-0003-4801-0761 \\ Ramón Alvarez' (iD 0000-0002-2505-4238 \\ Anunzziatta Fabruccini' (D) 0000-0001-7344-4751 \\ Fernando Massa² (iD 0000-0002-2922-4097
}

\section{Resumen}

Las enfermedades no transmisibles (ENT) comparten factores de riesgo conductuales y metabólicos con las enfermedades bucales. Ambas representan también un problema de salud pública.

Objetivo: determinar la prevalencia de caries, paradenciopatías y pérdida dentaria en personas que demandan atención en la facultad de odontología de la udelar.

Métodos: se relevó una muestra de los pacientes de la facultad de odontología, que respondieron un cuestionario sobre características sociodemográficas y hábitos vinculados a factores de riesgo comportamentales, se les realizaron mediciones antropométricas, de presión arterial, de glicemia capilar y un examen bucal.

Resultados: fueron encuestados 602 individuos, el cpod promedio fue de 16, la prevalencia de caries no tratada fue de 72, 8 \% y la prevalencia de periodontitis $42,9 \%$.

Conclusiones: Se recomienda la instalación de un programa preventivo-asistencial conjunto entre Facultad de Odontología y área salud de la UDELAR que integre la promoción y prevención de las enfermedades bucales y las no transmisibles.

Palabras clave: Enfermedades no transmisibles, Salud Bucal, Epidemiología, Interdisciplinario.

\footnotetext{
1 Servicio de Epidemiología y Estadística. Cátedra de odontología Social, Facultad de Odontología, Universidad de la República. serepistat@gmail.com

2 Instituto de Estadística, Facultad de Ciencias Económicas, Universidad de la República.
}

Fecha de recibido: 13/8/2020 - Fecha de aceptado: 27/1/2021 


\section{Abstract}

Noncommunicable diseases (NCDs) share behavioral and metabolic risk factors with oral diseases. Both also represent a public health problem.

Objective: to determine the prevalence of caries, paradenciopathies and tooth loss in people who demand attention at the udelar school of dentistry.

Methods: a sample of individuals who attend the school of dentistry answered a questionnaire on sociodemographic characteristics and habits together with behavioral risk factors. Anthropometric measurements, blood pressure, capillary glycemia were done, and an oral exam was made.

Results: 602 individuals were surveyed and the results showed that the average $\mathrm{dmft}$ was 16 , the prevalence of untreated caries was $72.8 \%$, and the prevalence of periodontitis was $42.9 \%$.

Conclusions: The installation of a joint preventive-care program between the Faculty of Dentistry and the health area of UDELAR that integrates the promotion and prevention of oral and non-communicable diseases is recommended.

Keywords: Non-communicable diseases, Oral Health, Epidemiology, Interdisciplinary.

\section{Introducción}

Las enfermedades no transmisibles (ENT) son un conjunto de patologías vinculadas al estilo de vida moderno que pueden explicarse al analizar el fenómeno de transición demoepidemiológica. Las ENT que generan la mayor carga de mortalidad a nivel mundial son fundamentalmente cuatro: enfermedades cardiovasculares (ECV), cáncer, enfermedades respiratorias crónicas y diabetes ${ }^{(1)}$. El $75 \%$ de las muertes por estas patologías se produce en

\section{Resumo}

As doenças não transmissíveis (DCNT) compartilham fatores de risco comportamentais e metabólicos com as doenças bucais. Ambos também representam um problema de saúde pública.

Objetivo: determinar a prevalência de cárie, paradenciopatias e perda de dente em pessoas que exigem atenção na faculdade de odontologia da udelar.

Métodos: foram avaliados 602 adultos, que responderam a um questionário sobre características sociodemográficas e hábitos relacionados a fatores de risco comportamentais, medidas antropométricas, pressão arterial, glicemia capilar e realização de exame oral.

Resultados: foram pesquisados 602 indivíduos com média de 16 cáries, prevalência de cárie não tratada de $72,8 \%$ e prevalência de periodontite de 42, 9\%.

Conclusóes: Recomenda-se a instalação de um programa de cuidados preventivos conjuntos entre a Faculdade de Odontologia e a área da saúde da UDELAR que integre a promoção e prevenção das doenças bucais e não transmissíveis.

Palavras-chave: Doenças não comunicáveis, Saúde Oral, Interdisciplinar.

países con ingresos medios y bajos. En Uruguay las ENT determinan casi 7 de cada 10 muertes registradas ${ }^{(2)}$.

La carga de enfermedades periodontales en un individuo con ENT puede exacerbar su desarrollo o su descompensación, mientras que el tratamiento de la enfermedad periodontal puede reducir la inflamación sistémica y mejorar el control glicémico en pacientes diabéticos o la disfunción endotelial en pacientes con ECV ${ }^{(3)}$. A su vez, la ausencia de tratamiento conduce a la pérdida de piezas dentales, deterioro del esta- 
do nutricional, así como una baja autoestima y calidad de vida.

En el caso de Caries, la alimentación también juega un rol importante: los azúcares refinados como la sacarosa en líquidos (refrescos, jugos, etc), la miel y las melazas empleadas en la industria de los alimentos industrializados aumentan el riesgo de su inicio como de su progresión. Mientras que el consumo de verduras, frutas y cereales integrales lo disminuyen. El consumo de frutas y verduras también ha mostrado beneficios en la prevención del cáncer oro faríngeo ${ }^{(4)}$. De acuerdo a la Segunda encuesta Nacional de Factores de Riesgo de ENT ${ }^{(5)}$ llevada a cabo por el Ministerio de Salud Pública de Uruguay, 50\% de la población adulta del país (25 a 64 años) tiene 3 o más factores de riesgo combinados.

En relación a la salud bucal, a partir de los datos provenientes del Primer Relevamiento $\mathrm{Na}$ cional realizado en Población joven y adulta uruguaya, año 2010-2011 (6), es posible afirmar que la prevalencia de Caries (CPOD mayor o igual 1) en jóvenes (15 a 24), adultos (35-44) y adultos mayores (65-74) es de 80,5\%, 99,5\% y 99,6 \%, respectivamente. El número promedio de dientes cariados, perdidos y obturados (CPOD) es de 4, 1, 15, 2 y 24, 1 para las edades de 15-24, 35-44 y 65-74 respectivamente $(7,6)$. La prevalencia de enfermedad periodontal (bolsas patológicas $\geq$ a $4 \mathrm{~mm}$ ) es de 11\%, 29\%, y $25 \%$ para la edades ya mencionadas ${ }^{(8,6)}$.

Si bien existen datos de la población uruguaya sobre las ENT y las enfermedades bucales, estos carecen de un enfoque común de factores de riesgo que tomen en cuenta ambas patologías. El presente trabajo tiene como objetivo determinar la prevalencia de factores de riesgo de las ENT y las enfermedades bucales más frecuentes (caries y paradenciopatías) en usuarios que demandan atención en la Facultad de Odontología de la UDELAR. Se presentan en este artículo los datos descriptivos correspondienes a salud bucal, aquellos correspondientes a ENT han sido ya publicados ${ }^{(9)}$.

\section{Metodología}

Se trató de un estudio descriptivo, transversal, realizado en el Servicio de Registro y Admisión de la Facultad de Odontología en el período agosto 2015 - mayo 2016.

La estrategia de muestreo utilizada tuvo en cuenta la estacionalidad de la concurrencia de las personas a registrarse en la Facultad de Odontología y los tres turnos en que funciona el Servicio de Registro (mañana, tarde y noche). Por tal motivo durante el período de recolección de datos el reclutamiento de los pacientes siguió una lógica de muestreo secuencial, donde participaban todos los pacientes que estaban citados en ese turno y en ese día. Por lo tanto dada esa logística, que el equipo de investigación consideró la más apropiada en términos de recursos humanos disponibles, se empleó un diseño mixto. El tamaño de muestra surge de considerar un diseño asimilable al Muestreo aleatorio Simple, que permite hacer estimaciones para prevalencias de hasta un $30 \%$ con una confianza del $95 \%$ y un error del 5\%, considerando hasta dos dominios de estimación ( sexo y tramo etario), siendo de tipo autoponderado. Esto implicó seleccionar 320 por dominio con lo cual fueron necesarios 640 pacientes. La metodología completa del estudio puede consultarse en el artículo previamente publicado que refiere al reporte de la prevalencia de las ENT en la población relevada ${ }^{(9)}$.

1) Recolección de datos:

Los participantes respondieron un cuestionario sobre aspectos sociodemográficos (sexo, edad, estudios cursados, ingresos mensuales, tipo de trabajo y cobertura asistencial en salud), factores de riesgo comportamentales y metabólicos. Además se registraron medidas antropométricas, glicemia capilar y la información referida a las patologías bucales (caries, paradenciopatías, lesiones de mucosa y pérdida dentaria).

Las patologías bucales fueron relevadas a través de un examen odontológico detallado. El mismo se realizó en sillón odontológico, con espejo, pinza y sonda CPI, se registró caries de corona y raíz, luego bolsa periodontal en todos 
los dientes presentes (manual OMS, 2013) ${ }^{(10)} \mathrm{y}$ pérdida de inserción por sextante.

3) Entrenamiento y Calibrado:

Se realizó una estandarización de criterios para la realización del cuestionario ( 4 reuniones conjuntas entre los diferentes profesionales participantes del estudio), finalmente se elaboró un instructivo de registro de las preguntas correspondientes a los formularios de investigación y se capacitó a los encuestadores para la utilización de las diferentes cartillas (alimentos, bebidas alcohólicas y actividad física).

Para el entrenamiento y calibrado de las patologías bucales, el equipo de trabajo tomó como referencia el Manual de Encuestas Básicas de la OMS ${ }^{(10)}$ del año 2013 y el Relevamiento $\mathrm{Na}$ cional de Salud Bucal realizado en Uruguay en el año $2011^{(6)}$; el mismo se realizó en el mes de marzo del año 2015 y participaron seis examinadores. Constó de 3 etapas: en una primera se estandarizaron los criterios de diagnóstico de tres de las patologías a relevar (caries, enfermedad periodontal y desgaste erosivo) para lo cual se contó con la colaboración de docentes de referencia pertenecientes a las disciplinas clínicas relativas a cada una de las patologías relevadas. En una segunda etapa de trabajo práctico-clínico, se realizó el entrenamiento para la condición de caries y enfermedad periodontal. Se realizó un estudio de calibración inter-examinador donde participaron seis examinadores y seis registradores. Se calculó el coeficiente kappa en cada una de las variables, en el caso de caries (corona) se obtuvo un valor de 0,85 , caries (raíz) el valor fue de 0,79 , en el caso del indicador CPI, se calibró bolsa periodontal y PIP, obteniéndose un valor de 0,71 y 0,79 respectivamente.

4) Variables e indicadores:

Para las patologías bucales, se consideraron los siguientes indicadores:

Caries: se utilizó el criterio de caries cavitada de la OMS (año 2013). Se utilizó el indicador de caries CPOD, para reportar la prevalencia \% $\mathrm{CPOD}>\mathrm{O}$, se calculó además el promedio de CPOD en la población y se utilizó el componente $\mathrm{C}$ del CPOD para reportar la caries no tratada.
Enfermedad periodontal: se reporta según el criterio del Índice periodontal comunitario (CPI) (dientes índice, OMS, 1997). Se define caso de periodontitis a la presencia de bolsa periodontal mayor a $4 \mathrm{~mm}$ y pérdida de inserción (PIP) mayor a $3 \mathrm{~mm}$ en alguno de los sextantes $^{(11)}$. El indicador utilizado fue la proporción de personas con periodontitis.

Pérdida dentaria: se presenta el promedio de dientes presentes por tramo etario, y la prevalencia de dentición no funcional. Se define dentición no funcional cuando la persona presenta menos de 20 dientes ${ }^{(12)}$.

Se resumieron las prevalencias de las variables cualitativas calculando proporciones mientras que para las variables cuantitativas se emplearon promedios. Se evaluó la incertidumbre de las estimaciones mediante intervalos de confianza al 95\%.

\section{Resultados}

\section{Caries dental}

La prevalencia de caries no tratada fue de $72,8 \%$, la mayoría de los tramos etarios presentaron una prevalencia similar, excepto en el tramo etario de 25-34 años que presentó la mayor prevalencia y el de 65-74 que presentó la menor $(82,7,44,1$ respectivamente). En cuanto al promedio de piezas dentarias que presentaron una lesión de caries no tratada se observan dos grupos, los primeros tres tramos etarios tienen un promedio que oscila en tres lesiones no tratadas, y los últimos tres tramos es menor a dos. Los grupos de 45-64 y 65-74 presentaron un incremento importante con respecto a los tres grupos más jóvenes.

Asimismo cuando se analiza el promedio de piezas afectadas por caries con respecto al promedio de piezas presentes, el valor resultante es de $17 \%$ para todas las edades (los valores fueron de $12 \%, 20 \%, 16 \%$ y $13 \%$, para las edades 15 a 24, 35-44, 45-64 y 65-74, respectivamente). En cuanto al CPOD se observó un aumento de los valores con la edad siendo el promedio de 16 piezas afectadas. 


\section{Enfermedad periodontal}

Respecto a la prevalencia de Periodontitis se aprecia una diferencia importante entre los valores correspondientes a los jóvenes (14,8\%) respecto a las otras edades consideradas que varían entre $38,1 \%$ y $66,7 \%$. Si bien la prevalencia aumenta con el incremento de la edad, esta no es monótona.

\section{Pérdida dentaria}

El 63, $6 \%$ de los encuestados presentó dentición funcional. Se constató que al aumentar la edad la prevalencia de la dentición no funcional se incrementa en forma monótona, menos en el último tramo ( $\geq 75$ ańos), observándose un incremento relevante en los tramos 45-64 y 6574 (Tabla 1).

\section{Lesiones de mucosa}

En cuanto a las lesiones de mucosa se encontró una prevalencia de 13\%. Entre ellas, las lesiones más frecuentes fueron: úlceras (34 casos), leucoplasia (25 casos) y candidiasis (22 casos).

Tabla 1: Distribución de a las patologías bucales en pacientes de Facultad de Odontología UdelaR.

\begin{tabular}{|c|c|c|c|c|c|c|c|}
\hline \multirow{3}{*}{ Edad } & \multirow{3}{*}{$\mathrm{N}$} & \multirow{3}{*}{$\begin{array}{c}\text { PERIODONTITIS } \\
\%(I C)^{*}\end{array}$} & \multicolumn{3}{|c|}{ CARIES } & \multicolumn{2}{|c|}{ PÉRDIDA DENTARIA } \\
\hline & & & $\begin{array}{c}\text { Caries No- } \\
\text { tratada }\end{array}$ & $\begin{array}{c}\text { Caries No- } \\
\text { tratada (Media, }\end{array}$ & CPOD & $\begin{array}{c}\text { Dentición no } \\
\text { funcional }\end{array}$ & $\begin{array}{c}\mathrm{N}^{\circ} \text { de dientes } \\
\text { presentes }\end{array}$ \\
\hline & & & $(\%, I C)$ & IC) & (Media, IC) & $(\%, I C)$ & (Media, IC) \\
\hline \multirow{2}{*}{ 15-24 años } & \multirow{2}{*}{88} & 14,8 & 71,6 & 2,93 & 6,02 & 1,1 & 23,987 \\
\hline & & $(7,3-22,2)$ & $(62,1-81,2)$ & $(2,19-3,68)$ & $(5,26-7,14)$ & $(0,0-3,4)$ & $(23,10-24,85)$ \\
\hline \multirow{2}{*}{ 25-34 años } & \multirow{2}{*}{104} & 42,3 & 82,7 & 3,56 & 11,42 & 8,7 & 19,78 \\
\hline & & $(32,8-51,8)$ & $(75,4-90,0)$ & $(2,87-4,24)$ & $(10,31-12,53)$ & $(3,2-14,1)$ & $(18,71-20,85)$ \\
\hline \multirow{2}{*}{ 35-44 años } & \multirow{2}{*}{114} & 38,1 & 79,8 & 3,04 & 16,43 & 33,3 & 14,93 \\
\hline & & $(29,1-47,0)$ & $(72,4-87,2)$ & $(2,42-3,67)$ & $(15,28-17,58)$ & $(24,6-42,0)$ & $(13,83-16,03)$ \\
\hline \multirow{2}{*}{ 45-64 años } & \multirow{2}{*}{210} & 51,5 & 72,9 & 1,95 & 20,8 & 51,7 & 11,09 \\
\hline & & $(44,5-58,5)$ & $(66,8-78,9)$ & $(1,64-2,26)$ & $(21,58-24,04)$ & $(44,8-58,6)$ & $(10,26-11,92)$ \\
\hline \multirow{2}{*}{ 65-74 años } & \multirow{2}{*}{59} & 66,7 & 44,1 & 1,22 & 22,17 & 77,8 & 9,7 \\
\hline & & $(54,0-79,4)$ & $(31,3-56,8)$ & $(0,74-1,70)$ & $(20,76-23,57)$ & $(66,6-89,0)$ & $(8,29-11,09)$ \\
\hline \multirow{2}{*}{$\geq 75$ años } & \multirow{2}{*}{27} & 47,8 & 70,4 & 1,78 & 24,18 & 76 & 7,52 \\
\hline & & $(27,0-68,1)$ & $(52,8-87,9)$ & $(0,60-2,95)$ & $(21,84-26,53)$ & $(58,9-93,1)$ & $(5,16-9,88)$ \\
\hline \multirow{2}{*}{ Total } & \multirow{2}{*}{602} & 42,9 & 72,8 & 2,5 & 16,33 & 36,4 & 14,9 \\
\hline & & $(38,9-46,9)$ & $(69,2-76,3)$ & $(2,26-2,74)$ & $(15,68-16,98)$ & $(32,6-40,3)$ & $(14,29-15,52)$ \\
\hline
\end{tabular}

\section{Discusión}

Se abordarán los tres tramos de edad recomendados por la OMS para comparación a nivel internacional: 15-24 (jóvenes), 35-44(adultos) y 65-74 (adultos mayores) ${ }^{(10)}$.

Tanto la prevalencia de periodontitis como la de caries no tratada fue significativamente ma- yor en los pacientes adultos que concurren a $\mathrm{Fa}$ cultad de Odontología demandando atención respecto al mismo rango etario a nivel nacional, lo cual era de esperarse ya que la población estudiada es precisamete la que demandaba atención en salud bucal.

Para el CPOD en jóvenes y adultos es mayor en FO que en los datos nacionales donde se regis- 
traron valores de 4, 15 (CI: 3, 8-4, 49) y 15, 2 (CI: 14, 24-16, 16) respectivamente $(6,7)$.

Cuando se utiliza el componente $\mathrm{C}$ del CPOD, en los jóvenes no es posible comparar la prevalencia de caries no tratada por no contar con datos publicados a nivel nacional pero seguramente por ser individuos que demandan atención en un centro asistencial se esperaría que fuera mayor. Para el caso de los adultos y adultos mayores, los valores del component $\mathrm{C}$ en relación a los del relevamiento nacional $(1,7$ (CI: 1,39-2,00) y 0, 66 (CI: 0.48-0.84), respectivamente) fueron significativamente mayores en la población que concurre a la Facultad de Odontología (7).

Con respecto a la periodontitis, en términos relativos la prevalencia aumenta un 157\% cuando pasamos del grupo de pacientes de 15-24 al 35-44 ańos $(66,7 / 14,8)$, mientras que aumenta un $75 \%$ cuando pasamos del grupo de pacientes de 35-44 años al de 65-74 años (66, $7 / 38,1)$. Cuando nos referimos a periodontitis de los adultos y los adultos mayores y los comparamos con la prevalencia a nivel nacional, 21,8 (CI: 17, 9-26, 3) ${ }^{(8)}$, observamos un incremento relativo del $140 \%(52,4 / 21,8)$ de esta condición en los pacientes que demandan atención en la Facultad de Odontología. Esto confirma que esta población presenta mayor carga de enfermedad.

Si analizamos la prevalencia de dentición no funcional de los pacientes que concurren a la Facultad comparados con los valores a nivel nacional en los tramos de 35-44 y 65-74 ${ }^{(12)}$ fueron simila- res; interpretándose que tal vez los pacientes que llegan a la Facultad no son aquellos con mayor proporción de pérdida dentaria porque llegan demandando restauraciones, terapia periodontal y prótesis parciales más que completas. Analizando la media de dientes presentes en boca los valores son similares entre los adultos mayores a nivel nacional $(9,73 \mathrm{CI}: 8,77-10,69)$, con respecto a los individuos que concurren a la Facultad (9, 7 CI: 8, 29-11: 09).

El hallazgo de mayor nivel de patología bucal en la población que concurre a Facultad, respecto a los datos nacionales coincide con el mayor nivel de patologias encontrado con respecto a las ENT a nivel naconal, recientemente publicado ${ }^{(9)}$.

\section{Conclusiones}

En cuanto a la prevalencia de las enfermedades bucales se comprobó que los individuos que llegan a la Facultad de Odontología presentan mayores niveles de patología de caries y periodontitis con respecto a la población uruguaya en general. Esto evidencia el rol social de la institución universitaria (siendo un prestador de salud a pesar de no integrar el SNIS) y la necesidad de diseñar programas integrales de prevención y promoción de la salud, que integren la prevención de ENT y enfermedades bucales. Resta aún estudiar a nivel individual la relación entre las enfermedades bucales y las ENT y sus factores de riesgo.

\section{Referencias}

1. WHO. Noncommunicablediseases. Factsheetupdated Junio 2018. [en linea] [ consultado 30 de octubre de 2018] Disponible en: http: //www.who.int/mediacentre/factsheets/fs355/en/

2. Uruguay. Ministerio de Salud Pública. Enfermedades No Transmisibles. [en línea] [ consultado 30 de octubre de 2018] Disponible en: http: //www.msp.gub.uy/noticia/enfermedades-no-transmisibles.

3. Eke PI, Wei L, Thornton-Evans GO, Borrell LN, Borgnakke WS, Dye B, Genco RJ. Risk indicators for periodontitis in US adults: NHANES 2009 to 2012. J Periodontol 2016 Oct; 87(10): 1174-85

4. Janket S-J, Baird AE, Chuang S-K, Jones JA. Meta-analysis of periodontal disease and risk of coronary heart disease and stroke. Oral Surg Oral Med Oral Pathol Oral Radiol Endod. 2003; 95(5): 559-569.

5. Uruguay. Ministerio de Salud Pública. Segunda Encuesta Nacional de Factores de Riesgo de Enfermedades No Transmisibles. PPENT. Montevideo, 2013. [en línea] [consultado 30 de octubre de 2018] 
Disponible en: http: //www.msp.gub.uy/publicaci $\% \mathrm{C} 3 \% \mathrm{~B} 3 \mathrm{n} / 2 \% \mathrm{C} 2 \% \mathrm{AA}$-encuesta-nacional-de-factores-de-riesgo-de-enfermedades-no-transmisibles.

6. Lorenzo-Erro S, Blanco S, Álvarez R, Massa F, Fabruccini-Fager A, Olmos P, Musto M, Ourens M. Primer Relevamiento Nacional de Salud Bucal de jóvenes y adultos uruguayos 2011. Facultad de Odontología. Universidad de la República. Montevideo, marzo 2018. Disponible: https: //www.colibri.udelar. edu.uy/jspui/handle/20.500.12008/18508

7. Álvarez L, Liberman J, Abreu S, Mangarelli C, Correa MB, Demarco FF, Lorenzo S, Nascimento GG.Dental caries in Uruguayan adults and elders: findings from the first Uruguayan NationalOral Health Survey. Cad Saude Publica. 2015 Aug; 31(8): 1663-72. doi: 10.1590/0102-311X00132214.

8. Lorenzo SM, Álvarez R, Andrade E, Piccardo V, Francia A, Massa F, Correa MB, Peres MA. Periodontal conditions and associated factors among adults and the elderly: findings from the first National Oral Health Survey in Uruguay.Cad Saude Publica. 2015 Nov; 31(11): 2425-36. doi: 10.1590/0102$311 \mathrm{X} 00012115$.

9. Lorenzo-Erro SL, Skapino E, Musto M, Olmos P, Álvarez R, Fabruccinni A, Massa F, Moliterno P, Piovesán S, Cosetti L, Pisón F. Salud Bucal y Enfermedades no transmisibles en pacientes de un centro de enseñanza terciaria, Montevideo, Uruguay 2016. Parte 1. Odontoestomatología. 2020; 22(36).

10. World Health Organization. Oral Health Surveys. Basic Methods. 5th Edition [Internet].Geneva; 2013. 11. Vettore MV, Marques RA, Peres MA. [Social inequalities and periodontal disease: multilevel approach in SBBrasil 2010 survey]. RevSaudePublica. 2013; 47Suppl 3: 29-39.

12. Nicola-Laguzzi P, Silveira-Schuch H, Derrégibus-Medina L, Rodríguez-de Amores A, Demarco FF, Lorenzo S. Tooth loss in the elderly in Uruguay. J Public Health Dent 2016; 76: 143-151. doi: 10.1111/jphd.12123

\section{Fuente de financiamiento:}

\section{Llamado I+D CSIC}

\section{Aprobación Comité de Ética:}

Aprobado por el comité de ética de Facultad de Odontología (Expediente nº 091900000121-14).

\section{Nota declaración de interés:}

Los autores declaran no tener conflicto de intereses.

\section{Nota contribución de los autores:}

1. Concepción y diseño del estudio

2. Adquisición de datos

3. Análisis de datos

4. Discusión de los resultados

5. Redacción del manuscrito

6. Aprobación de la versión final del manuscrito

SMLE ha contribuido en: 1, 3, 4, 5 y 6 .

RA ha contribuido en: $1,3,4$ y 6 .

AF ha contribuido en: 2, 3, 4, 5 y 6 .

FM ha contribuido en: 3,4 y 6.

\section{Nota de aceptación:}

Este artículo fue aprobado por la editora de la revista Mag. Dra. Vanesa Pereira-Prado. 